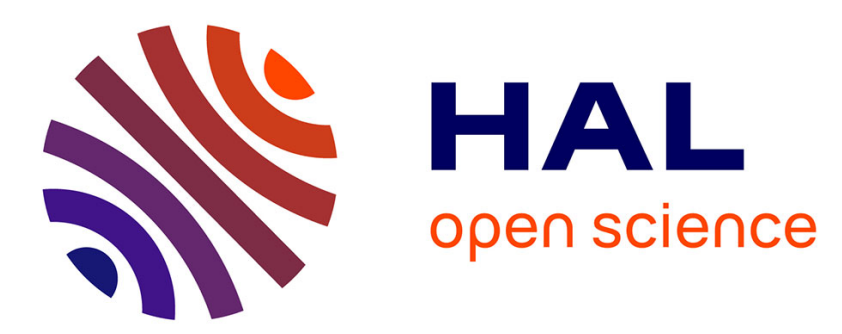

\title{
Assessment of measurement performance for a low field side IDTT plasma position reflectometry system
}

F da Silva, J Ferreira, J Santos, Stéphane Heuraux, E Ricardo, G de Masi, O

Tudisco, R Cavazzana, O D'arcangelo

\section{- To cite this version:}

F da Silva, J Ferreira, J Santos, Stéphane Heuraux, E Ricardo, et al.. Assessment of measurement performance for a low field side IDTT plasma position reflectometry system. Fusion Engineering and Design, 2021, 168, pp.112405. 10.1016/j.fusengdes.2021.112405 . hal-03164339

\section{HAL Id: hal-03164339 \\ https://hal.univ-lorraine.fr/hal-03164339}

Submitted on 9 Mar 2021

HAL is a multi-disciplinary open access archive for the deposit and dissemination of scientific research documents, whether they are published or not. The documents may come from teaching and research institutions in France or abroad, or from public or private research centers.
L'archive ouverte pluridisciplinaire HAL, est destinée au dépôt et à la diffusion de documents scientifiques de niveau recherche, publiés ou non, émanant des établissements d'enseignement et de recherche français ou étrangers, des laboratoires publics ou privés. 


\title{
Assessment of measurement performance for a low field side IDTT plasma position reflectometry system
}

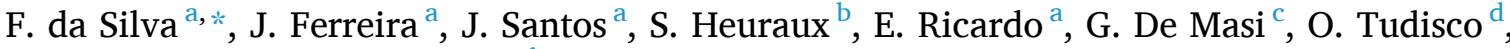 \\ R. Cavazzana ${ }^{c}$, O. D’Arcangelo ${ }^{\mathrm{d}}$ \\ ${ }^{a}$ Instituto de Plasmas e Fusão Nuclear, Instituto Superior Técnico, Universidade de Lisboa, 1049-001 Lisboa, Portugal \\ b Institut Jean Lamour, UMR 7198 CNRS-Université de Lorraine BP 50840, F-5401 Nancy, France \\ ${ }^{c}$ Consorzio RFX, 35127 Padova, Italy \\ ${ }^{\mathrm{d}}$ Associazione EURATOM-ENEA-CR Frascati, 00044 Frascati, Italy
}

Keywords:

FDTD

IDTT

Reflectometry

Numerical simulation

DEMO

\begin{abstract}
A B S T R A C T
The Italian Divertor Test Tokamak (IDTT) facility will study advanced exhaust solutions applicable to DEMO. This new machine also opens the possibility to test and validate relevant non-magnetic control diagnostics in support of a DEMO design implementation. Reflectometry, compatible with a full grade reactor implementation, has been proposed as a source of real-time (RT) plasma position and shape measurements for control purposes, in replacement or complement of standard magnetic measurements, to ensure reliability and safety on the machine. Additionally, such a system can be useful to obtain the characteristics of edge turbulence, useful for Neoclassical Tearing Mode (NTM) control or to improve the efficiency of other diagnostics as Collective Thomson Scattering (CTS). This new control technique based on multiple, poloidally distributed, non-magnetic measurements, must be tested, in all of its aspects, before it can be fully implemented in future fusion reactors. IDTT will be one of the best candidates to implement and build a knowledge database of nonstandard reflectometry (away from the equatorial plane) that will be needed on DEMO and is currently unavailable. The performance of three Ordinary mode (O-mode) Plasma Position Reflectometers (PPR) at the Lower Field Side (LFS) on IDTT is assessed using the two-dimensional (2D) full-wave Finite-Difference Time-Domain (FDTD) code, REFMULF, in two of the foreseen IDTT plasma scenarios: 5MA Single Null (SN) and Double Null (DN) configurations.
\end{abstract}

\section{Introduction and objectives}

The Italian Divertor Test Tokamak (IDTT) facility is a new machine proposed to study advanced exhaust solutions in conditions approaching as much as possible those planned for DEMO. ITER will operate in a detached condition, having a radiating volume in front of the divertor [1]. However, this solution may prove inadequate, and consequently, the need for sound alternatives for DEMO is felt. The access and available space on the Low Filed Side (LFS) open the possibility to test and validate relevant non-magnetic control diagnostics, in reactor like regimes, in support of a DEMO design implementation.

As in all fusion machines, there will be a need for diagnostics to gather knowledge about the physical processes occurring in the plasma, for engineering purposes and control fundamental to the safety of the machine and to assure the reliability and redundancy required to maintain a steady state plasma [2]. Reflectometry has become one of the most important techniques to diagnose fusion plasmas. For more than 30 years, since its first trials both in O-mode [3] and X-mode [4] reflectometry has had a continuous influx of developments based on strong theoretical developments, in the interpretation of plasma turbulence [5, 6] and the impact it has on measurements such the enlargement of the probing beam and consequent loss in resolution [7], in hardware developments such as fast systems [8] which have an impact on the type and quality of measurements possible [9], the improvement on the understanding of phenomena as the effects on nonlinear responses in Doppler reflectometry [10] or the expansion of the capabilities of reflectometry opening the possibility of reconstruct hollow areas in density profiles [11], an accomplish deemed not possible in the early years of the technique and a sustained effort on the improvement of processing algorithms [12]. Advantages such as versatility, multi-usage, neutron compatible front face components, ultra-fast operation make its use foreseen in the forthcoming generation of machines $[13,14]$ such as 
ITER and DEMO [15]. Ordinary mode (O-mode) reflectometry, due to its compatibility with a full grade reactor implementation, was proposed as a source of real-time (RT) plasma position and shape measurements for control purposes, in replacement or complement of standard magnetic measurements and successfully tested on ASDEX Upgrade both in the LFS and High Field Side (HFS) [16,17]. On DEMO, the foreseen placement of magnetic sensors behind the thick blanket modules will introduce an inherent delay in the corresponding measurements, possibly preventing them from being used in fast control feedback loops. This means that a fast position and shape feedback controller must make use of alternative multiple, poloidally distributed, non-magnetic measurements such as the ones provided by O-mode reflectometry. This new control technique must be tested, in all its aspects, before it can be fully implemented in future fusion reactors. Also, for IDTT, this diagnostic can be a useful asset, providing edge electron density profile information namely in the detachment region at lines-of sight (LOS) closer to the divertor. Therefore, assessing the different applications that could be deployed on IDTT is of major importance.

This paper assesses the performance of a set of 3 plasma position reflectometers (PPR) at the IDTT. This assessment was carried out using the two-dimensional (2D) full-wave Finite-Difference Time-Domain (FDTD) Reflectometry Simulator (REFMULF) in two of the foreseen IDTT plasma scenarios: 5MA Single Null (SN) and Double Null (DN) configurations, at the Start Of Flattop (SOF), $T=36 \mathrm{~s}$, that is at the start of the flattop of the plasma current. These scenarios are the most common ones and constitute a consistent starting point for the assessment of the possibility of having one or more PPR systems at IDTT.

The IDTT Interim Design Report (IDR), dating from April of 2019, is scarce on information concerning the reflectometry systems previewed for the machine. Therein, two reflectometers have been described: one operating in Extraordinary Mode (X-mode) and another in Ordinary Mode (O-mode) [18]. The PPR system we put forward is not related with those systems but rather an alternative one that can complement and eventually be merged into IDTT reflectometry tentative plans.

The workflow for the present study can be broadly divided in:

- Select the data applicable to the PPR system simulations from the input data (plasma scenarios) provided in [19] and [20], verifying the quality of the data and identifying any missing data.

- Set-up of three synthetic reflectometers, three on the Low Field Side (LFS), the first (gap $0^{\circ}$ ) on the equatorial plane, the second on the upper torus midplane (gap $45^{\circ}$ ) and the third on the lower torus midplane (gap $-60^{\circ}$ ). See Fig. 1 for a location of the synthetic diagnostics around the torus.

- Assessment of the behavior of the reflectometers of gaps $0^{\circ}, 45^{\circ}$ and $-60^{\circ}$ under a relevant IDTT scenario to characterise the type of signals that can be expected and the signal processing techniques required, namely the amplitude and frequency dynamic ranges in the PPR operating frequency range (from $18 \mathrm{GHz}-95 \mathrm{GHz}$ or $18 \mathrm{GHz}-105 \mathrm{GHz}$, depending on the scenario).

- Assessment of the effects associated with the structures surrounding the antennas on the measurements of the reflectometers at each gap.

- Assessment of the impact of probing the plasma away from the midplane, where the iso-density lines exhibit poloidal divergence and curvature, as is the case of gaps $45^{\circ}$ and $-60^{\circ}$.

In the remainder of the paper we will describe, in Section 2, how the problem has been modeled, with the description how the input data was handled, the options taken to prepare the synthetic diagnostics and the way simulations were setup. In Section 3 we present the results, explaining the methods used to obtain them. The conclusions and plans for further work are discussed in Section 4.
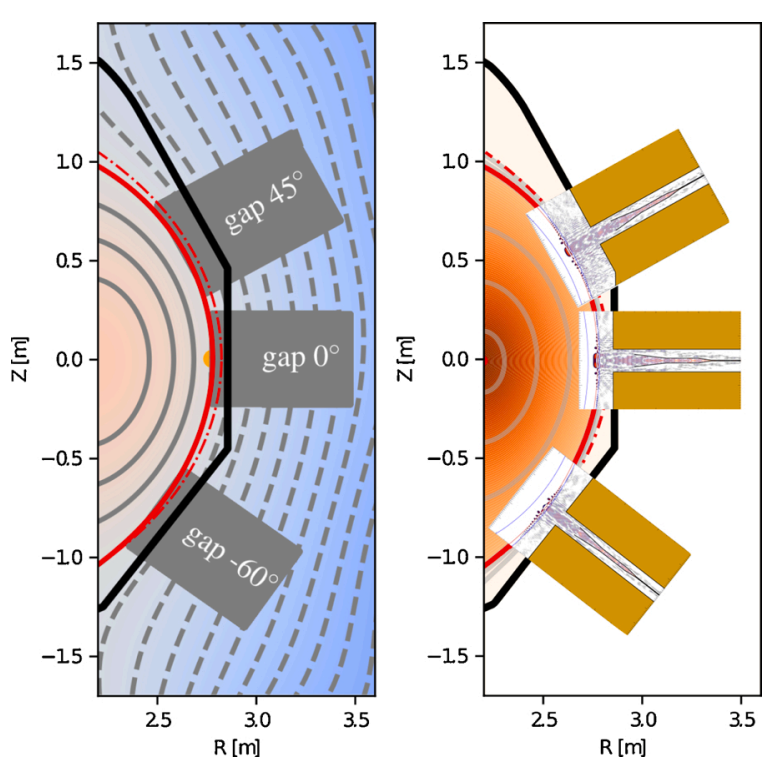

Fig. 1. Representation of the IDTT vessel with the three locations selected for the implementation of the synthetic reflectometers. Starting from the top of the vessel and moving clockwise we identify gaps $45^{\circ}, 0^{\circ}$ and $-60^{\circ}$. On the left we can see separatrices corresponding to the different scenarios foreseen for IDTT. On the right, the magnetic flux poloidal surface for SN scenario with the separatrix marked in red. Representation of the IDTT vessel showing, for the SN scenario, on the left, the 2D map of the magnetic flux in machine coordinates $\Psi(R, Z)$ and, on the right, the obtained 2D map of the electronic density in machine coordinates $n_{e}(R, Z)$. The continuous red line denotes the magnetic separatrix for SN. Also represented is the magnetic separatrix for the DN, in a red dash-dot, for comparison.

\section{Modelling}

\subsection{Input data}

The planned reference scenario for IDTT is a SN scenario at 5.5 MA [21]. After reviewing the available data, we selected the SN and DN scenarios as being the most relevant to perform the simulations, since they correspond to plasma scenarios expected to be used from the start of IDTT operation and therefore allowing the assessment of the reflectometry measurements right from the tokamak's first plasmas. As the PPR systems are required to provide their main function on the flat-top phase of the discharges, the simulations were performed at the flat-top phase of the plasma current $\left(I_{p}\right)$, in particular, at the time instant $T=$ $36 \mathrm{~s}$, the Start Of Flattop (SOF), that is at the start of the flattop of the plasma current.

While assessing the provided input data, we identified two main shortcomings common to all datasets: (i) the lack of information below the separatrix density, in the so-called Scrape-Off Layer (SOL) region $\left(\rho_{\mathrm{pol}}>1, R>R_{s}\right)$, and (ii) the low resolution of the 2D magnetic configuration data, when compared with the description required to run a FDTD code. The low resolution of the data could not be avoided, and we had to interpolate the data. The lack of SOL data was more difficult to overcome. Since the measurements performed by the PPR system will be made mostly in this region (see Fig. 2 - top), we chose to extrapolated the SOL data from the available core plasma data using a model. At the start time of this work, a description of the SOL electron density, fully consistent with the given EQDSK equilibrium, was not available. Therefore, an educated guess model had to be implemented. The pursued solution was based on the density profiles appearing in [18] using a fit on the extracted data with the expression proposed in [22]

$n_{e}^{\text {sol }}(x)=h_{\text {plat }}+\frac{h}{2}\left[1+\frac{\left(1+s^{\text {core }} x\right) \mathrm{e}^{x}-\left(1+s^{\text {sol }} x\right) \mathrm{e}^{-x}}{\mathrm{e}^{x}+\mathrm{e}^{-x}}\right]$ 

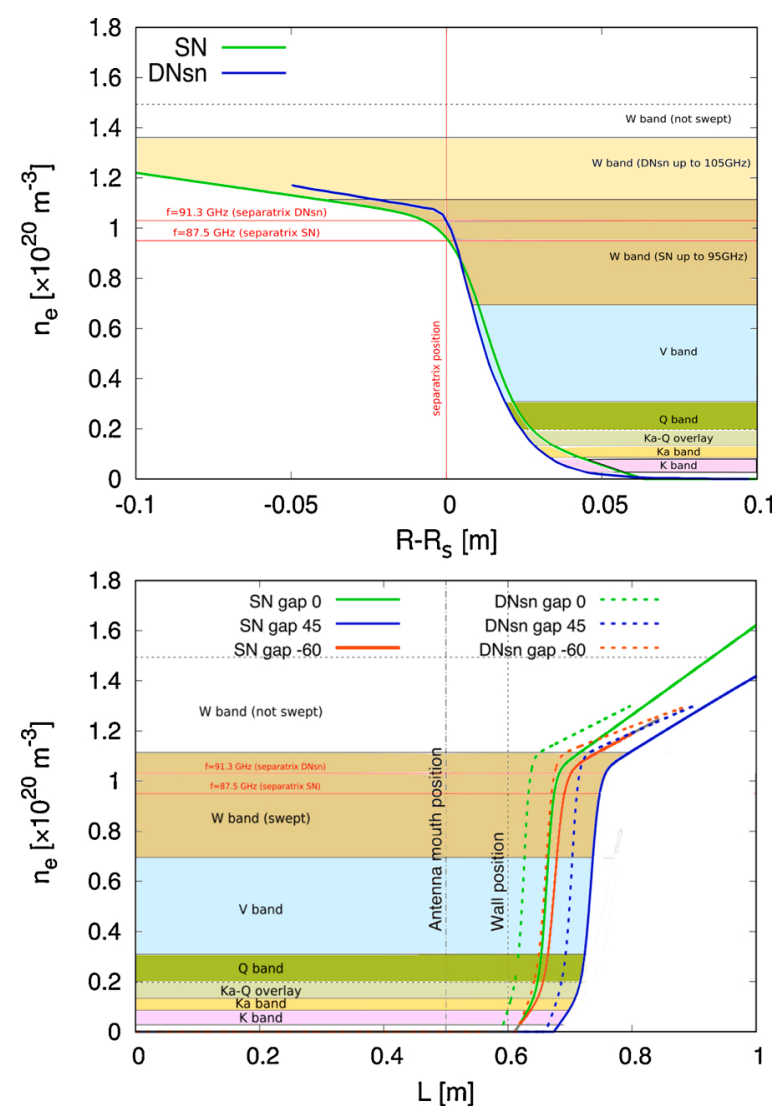

Fig. 2. Density profiles from $\mathrm{SN}$ and $\mathrm{DN}$ scenarios in real space $R-R_{s}$ at the midplane (top) and the same two profiles mapped in the length coordinate, $L$, for each gap (bottom).

with $x=\frac{p_{\mathrm{pos}}-r}{2 w_{r}}$

where $s^{\text {core }}$ and $s^{\text {sol }}$ are coefficients that better fit the inner and outer linear shape away from the pedestal, $r$ is the radius, $h$ is the pedestal height, $w_{r}$ is the pedestal width, $p_{\text {pos }}$ is the pedestal position along a LOS, and $h_{\text {plat }}$ the first density step. For the SN scenario the fit results in

$$
\begin{aligned}
s_{\text {core }} & =5.0 \times 10^{-2}\left[\mathrm{~m}^{-1}\right], s_{\text {sol }}=-0.11141448\left[\mathrm{~m}^{-1}\right], \\
h & =8.1431 \times 10^{19}\left[\mathrm{~m}^{-3}\right], p_{\text {pos }}=0.01234699[\mathrm{~m}], \\
w_{r} & =0.00568111[\mathrm{~m}], h_{\text {plat }}=2.0475 \times 10^{19}\left[\mathrm{~m}^{-3}\right],
\end{aligned}
$$

while for the DN

$$
\begin{gathered}
s_{\text {core }}=0.02845143\left[\mathrm{~m}^{-1}\right], s_{\text {sol }}=-0.16865337\left[\mathrm{~m}^{-1}\right], \\
h=8.0460 \times 10^{19}\left[\mathrm{~m}^{-3}\right], p_{\text {pos }}=0.00876321[\mathrm{~m}], \\
w_{r}=0.00456687[\mathrm{~m}], h_{\text {plat }}=2.8111 \times 10^{19}\left[\mathrm{~m}^{-3}\right] .
\end{gathered}
$$

The selected scenarios, complemented by the extrapolated SOL data, and the CAD inner-vessel geometry and separatrix/magnetic distribution models, available in [19] and [20], were used to set-up in REFMULF the synthetic Ordinary Mode (O-mode) plasma position reflectometers corresponding to the selected three gaps/lines-of-sight (LOS). The simulations performed using these synthetic diagnostics allowed us to: (i) estimate the measurement performance of the proposed systems, and (ii) verify if the PPR system will be able to fulfill the error requirements within an error inferior to $\pm 1 \mathrm{~cm}$, even in adverse measurement conditions of operation and/or other non-contemplated design constraints $[23,24]$. The result of mapping the reference profiles to the three simulated LOS is illustrated in Fig. 2. The top plot shows the electronic densities for SN and DN scenarios on radial flux coordinates mapped on $R-R_{s}$, being $R_{s}$ the position of the separatrix at $\rho_{\text {pol }}=1$. The profiles exhibit a core region with a relatively linear shape density, followed by a sharp drop-off region. The SOL density drops to zero before the position of the first wall. The red vertical line indicates the position of the magnetic separatrix $\left(\rho_{\text {pol }}=1\right)$ while the horizontal ones, the density value at the separatrix for each of the scenarios. The densities covered by each of the PPR reflectometer bands are also represented by the multi-colored shaded regions.

\subsubsection{D Density maps}

Two dimensional (2D) poloidal maps of the magnetic reconstruction are used to map the density profile model adopted into a $2 \mathrm{D}$ reconstruction of the electronic density $n_{e}$ in the machine frame of reference. IDTT plasma equilibrium data is provided in the form of equilibria EQDSK files, which allows data profiles to be mapped into 2D space, such as the fitted density profiles described before. Given a physical quantity as a function of real space along a line of sight, $n_{e}\left(r_{\mathrm{LOS}}\right)$, or as a function of a radial flux coordinate, $n_{e}\left(\rho_{\mathrm{pol}}\right)$, with $\rho_{\mathrm{pol}}=\sqrt{\Psi / \Psi_{\mathrm{LCFS}}}$, commonly known as the normalized poloidal-flux radius, and $\Psi_{\mathrm{LCFS}}$ being the poloidal flux at the last closed flux surface (LCFS), and having the $2 \mathrm{D}$ maps of the flux in machine coordinates $(R, Z)$, assuming a toroidal invariance, the profiles can be mapped into the machine coordinates, scalable with the coordinate system of the REFMULF code:

$n_{e}\left(r_{\mathrm{LOS}}\right) \stackrel{\operatorname{Los}_{\mathrm{LO}}\left(\rho_{\mathrm{pol}}\right)}{\longrightarrow} n_{e}\left(\rho_{\mathrm{pol}}\right) \stackrel{\rho_{\mathrm{pol}}(R, Z)}{\longrightarrow} n_{e}(R, Z)$.

The accuracy and completeness of this mapping depends on the accuracy and completeness of $\Psi(R, Z)$ and $n_{e}\left(\rho_{\text {pol }}\right)$, and, being the primary inputs for the 2D full-wave microwave simulations, they will directly impact their results. Concerning the FDTD simulations, for a given frequency $f$, the required spatial resolution is a fraction of the vacuum wavelength, $\delta_{r}=\lambda_{0} / N$, where $N$ is the number of points per wavelength. The higher the value of $N$, the lower the phase error committed in the simulations. For REFMULF, a typical choice of $N=20$ requires a point density of $1767 \mathrm{pts} / \mathrm{m}$ at the lower $\mathrm{K}$ band, which increases to about $7353 \mathrm{pts} / \mathrm{m}$ for the higher band W. Therefore, we have resorted to data interpolation in order to achieve the required grid resolution. This results in big simulation domains, for instance in the gap $0^{\circ}$, the smallest gap simulated, for SN scenario, the $\mathrm{K}$ band has an extension of $1348 \times$ 899 points for the $\mathrm{K}$ band and $7863 \times 5242$ in the upper band $\mathrm{W}$.

As seen in the previous sections, we need the 2D map for the electron density $n_{e}$ as a function of the machine coordinates, that is $n_{e}(R, Z)$. Therefore, we took the 1D model of the electron density given in Eq. (1) where the density is modelled as a function of the real space along LOS at the midplane, and use the 2D maps of the poloidal magnetic flux in machine coordinates $\Psi(R, Z)$ to get $n_{e}(R, Z)$ as per Eq. (2). The result from this procedure, for the SN scenario is illustrated in Fig. 1, which shows, on the left, the 2D map of the magnetic flux in machine coordinates $\Psi(R, Z)$ and, on the right, the obtained 2D map of the electronic density in machine coordinates $n_{e}(R, Z)$.

To perform the simulations for gaps $0^{\circ}, 45^{\circ}$ and $-60^{\circ}$, it is not necessary to use the electron density all over the plasma chamber, being enough to have this information in the vicinity of the antennas, in the region where the probing waves are expected to propagate and reflect back. Therefore, we selected Regions of Interest (ROI) for each gap, which are wide enough to include all the physics required to describe the scenario and reflectometer setup such as multi-reflections. These can be seen in Fig. 1. To achieve the necessary higher resolution, we interpolated the 2D density map in the ROI; the number of points after interpolation depends on the frequency band and increases from the $\mathrm{K}$ band $(18-26.5 \mathrm{GHz})$ to the $\mathrm{W}$ band $(75-95 \mathrm{GHz}$, for $\mathrm{SN}$ or $75-105 \mathrm{GHz}$, for $\mathrm{DN})$, since the wavelength decreases with frequency, as seen in 2.1.1. For a given scenario and SOL model, the density profiles measured by the systems of gaps $0^{\circ}, 45^{\circ}$ and $-60^{\circ}$, when expressed at the midplane or in radial flux coordinates, are the same. However, when expressed in length coordinates, the profiles along the LOS facing each of the gaps exhibit very different shapes, which are due to the fact that the 
configuration of the magnetic flux in the vicinity of each gap is different, resulting in a different density profile remapping. This is illustrated in Fig. 2, where the profiles for SN and DN scenarios are depicted both in real space at the midplane coordinate, (top), easily mapped to radial flux coordinates, and in the length coordinate, $\mathrm{L}$, (right), showing that the exact same profiles in radial flux coordinates correspond to very different profiles being probed by each gap along their LOS.

\subsection{Emission structures}

The antennas and the accesses to the vessel for reflectometry were not, at the start of this work, yet defined. Therefore, we adopted one of the antenna designs being used in the simulation and design of the DEMO PPR, which can be observed at the top of Fig. 3. The transmission (Tx) and reception $(\mathrm{Rx})$ antennas are standard horns, place side-by-side, having the H-plane in a poloidal plane of the machine. Their lines of sight (LOS) are parallel. This forms a bistatic configuration with toroidally distributed antennas. The antennas access the plasma through a circular opening and are $10 \mathrm{~cm}$ recessed in reference to the wall for thermal and erosion protection. To pass from a three dimensional reality to a 2D simulation model we used the approach described in [25], which has been used in ITER [26] and DEMO [15] 2D simulations. In the 2D simulations, the 3D antenna distribution is approximated, considering that the two 2D H-plane cuts for the Tx and Rx antennas collapse on a single $2 \mathrm{D} \mathrm{Tx} / \mathrm{Rx}$ plane. The simulation of this mono-static setup is possible due to the use of a Unidimensional Transparent Source (UTS)
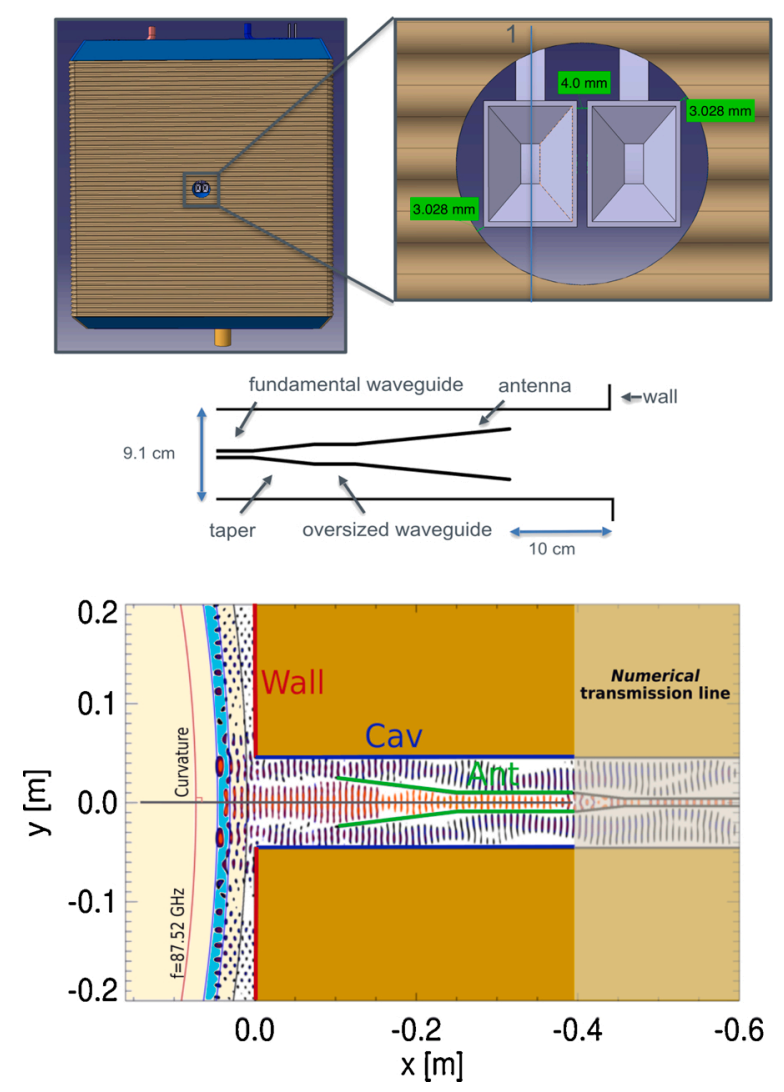

Fig. 3. On top, the CAD of one of the antenna models used in the simulations of DEMO and adopted for the present work. The 2D schematic of the antenna can be seen below. On the bottom one observes a snapshot of the synthetic reflectometers of gap $0^{\circ}$ for Ka band operating at a frequency of $40 \mathrm{GHz}$. The blueshaded region indicates the iso-density region corresponding to the frequency limits of the Ka band. The shaded part between $[-0.4,-0.6][\mathrm{m}]$ in the simulation box provides a termination to the antenna giving a boundary to the oversized antenna and waveguide numerically equivalent to a well behaved transmission line.
[27], which decouples wave emission and wave reception as it is in a bistatic set-up assuming that the emitter and receiver have the same design. The compromise done when using a 2D model is further discussed in [25]. An additional compromise to have in mind is that any modes forming within the circular access will have a structure proper to a circular oversized waveguide while the $2 \mathrm{D}$ representation of the opening has a rectangular $2 D$ structure. At the bottom of Fig. 3 we see a snapshot of the synthetic reflectometers of gap $0^{\circ}$ for Ka band at a frequency of $f=40 \mathrm{GHz}$. The blue-shaded region indicates the iso-density region corresponding to the frequency limits of the Ka band, while the solid black line indicates the iso-density corresponding to a frequency of $f=87.52 \mathrm{GHz}$, the cut-off frequency of the separatrix for the SN scenario. For each of the 3 gaps considered, the LOS of the antenna was designed to be perpendicular to the separatrix of the SN scenario. With the DN scenario the same line of sight (SN optimised) was used and being referred in this work as Double Null with SN optimisation (DNsn). The system covers a very large frequency range divided in five standard microwave bands, K $(18-26.5 \mathrm{GHz}), \quad \mathrm{Ka} \quad(26.5-39.5 \mathrm{GHz}), \quad \mathrm{Q}$ (33-50 GHz), V (50-75 GHz) and W (75-95 GHz, for SN or 75-105 GHz, for $\mathrm{DN}$ ). Since the same physical antenna and waveguide are used for all the bands, their electric dimensions vary substantially. While for the $\mathrm{K}$ band the waveguide dimension corresponds to a fundamental waveguide, as frequency augments throughout the bands, the waveguide becomes increasingly oversized. The antenna becomes further directive with frequency as well. The implementation of such a system is a demanding technical task but perfectly within the state of the art. A mockup of gaps 6 and 4, which had been previewed for ITER, covering bands $\mathrm{K}$ to $\mathrm{V}$, has been successfully build and tested $[28,29]$.

\subsection{Synthetic reflectometers and simulation setup}

The synthetic O-Mode Frequency-Modulated Continuous-Wave (FMCW) broadband reflectometers of gaps $0^{\circ}, 45^{\circ}$ and $-60^{\circ}$ were implemented with REFMULF. This code is part of the REFMUL* family of codes developed by IPFN, IST. It is a 2D code able to cope with full polarization waves, treating all components of the electric, E, and magnetic, $\mathrm{H}$, fields of the wave and supports a generic external magnetic field $\mathrm{B}_{0}$, coupling the Transverse-Electric Mode (TE, X-mode) with the Transverse-Magnetic Mode (TM, O-mode) via a linear vector differential equation for $\mathrm{J}$, which is solved using a modified Xu-Yuan kernel [27] [30], with extended long-run stability. The components of the external magnetic field $\mathrm{B}_{0}$ lying on the propagation plane are responsible for linking the TE and TM modes, enabling the description of oblique propagation. For a $\mathrm{B}_{0}$ purely perpendicular to the propagation plane, the code describes simultaneously $\mathrm{O}$-mode and $\mathrm{X}$-mode propagation. A description of the code, including its most relevant references, is provided in [31].

The synthetic reflectometers model both the specific location within the IDTT vacuum vessel and the specific geometry of the plasma access at each gap. Simulations were performed using five standard microwave bands $\mathrm{K}, \mathrm{Ka}, \mathrm{Q}, \mathrm{V}$ and $\mathrm{W}$, covering a frequency range of [18-95 GHz] for the SN scenario and [18-105 GHz] for the DN scenario. The plasma is modelled, according to the selected magnetic configuration, as described in the previous sections. The emission and reception structures of each system were modelled according to the CAD drawings of IDTT made available in [19] and [20]. These structures were modelled as Perfect Electric Conductor (PEC) surfaces and comprise the recessed antennas, the opening through which the antennas access the plasma and including the first-wall surface.

During simulations, a FMCW signal is excited at the antenna with frequencies ranging from the minimum to the maximum frequency of each band. The probing beam propagates to the plasma being reflected back to the antennas where is decoupled from the emission through the use of a UTS [27]. The UTS decoupling allows simulating both the emission and reception antennas in 2D. The plasma input in the synthetic diagnostic is the (interpolated) ROI corresponding to the 
frequency band being simulated. A Perfectly Matched Layer (PML) surrounds the simulation box defined by the ROI so that the electromagnetic waves reaching its boundaries are not reflected back into the simulation domain, simulating in practice a free propagation outside the ROI [32]. In Fig. 3, bottom, the PEC surfaces are divided into the antenna (green), the space where the antennas are installed (blue), and the wall surfaces (red). We can observe the contours of the electric field structure formed by the superposition of the emitted and reflected beams as well as the curvature and tilt exhibited by the plasma relative to the optical axis of the antenna.

Simulations were organised according to the description of the propagating medium considered and with the emission/reception structures used.

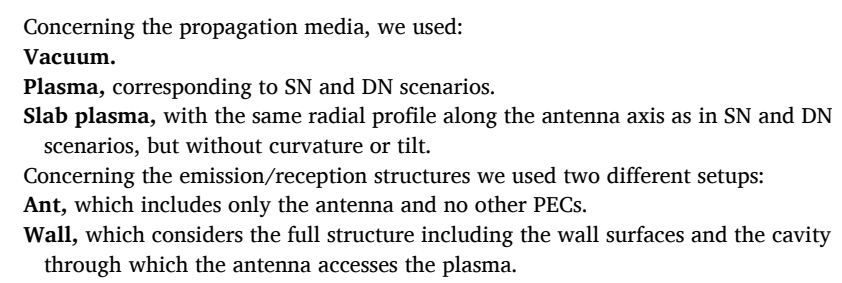

Any combination of media and emission/reception structures can be used in the simulations, which is helpful to identify different problems. The simulations performed with vacuum were used to characterise the antennas, namely the radiated beam (radiation pattern), and how the surrounding structure affects it. The simulations performed with slab plasmas were used to obtain reference cases against which the performance of the different gaps is evaluated, in particular the measurement error. The considered slab plasmas have the same radial profile along the antenna axis as in scenarios SN and DN but do not exhibit any curvature or tilt. Therefore, using only the antennas, we are essentially simulating standard FMCW reflectometry measurements, which is a diagnostic technique that was already demonstrated to be valid for plasma position measurements [16]. Moreover, comparing the simulations for plasma scenarios SN and DN, which include curvature and tilt, against the ones performed with the slab plasmas, we can assess the effect of those plasma characteristics in the measurements. In the profile reconstruction, the initialisation was considered a solved problem, see [33]. In Fig. 4, we show two snapshots of gap $0^{\circ}$ simulations performed with a slab plasma corresponding to plasma $\mathrm{SN}$ scenario, taken during the sweeps of the bands $\mathrm{K}$ (top) at a frequency of $f=26.5 \mathrm{GHz}$ and $\mathrm{W}$ (bottom) at $f=95 \mathrm{GHz}$. The vertical blue lines mark the iso-density region corresponding to the limits of the bands; the red line shows the position of the slab separatrix corresponding to a frequency of $f=$ $87.52 \mathrm{GHz}$, which falls within the limits of the $\mathrm{W}$ band. This setup, using slab plasma and just the antenna (Ant), is used to judge how all the other cases deviate from the standard reference case. We see that the field structure is symmetric in relation to the antenna axis and that reflection occurs in a spot in front of the antenna as can be observed by the swelling of the field, in a scale compatible with the Airy wavenumber $[34,35]$.

\section{Results}

\subsection{Assessment of the antenna characteristics}

The free-space radiation characteristics of pyramidal horn antennas, like the ones used in the PPR in-vessel systems, is well known and documented in the literature [36]. However, when such antennas are installed in the small access perforation, recessed into the wall, the original radiation characteristics are modified $[37,28]$. In this subsection, we look at the influence of the wall modules on the radiation properties of the antennas in the studied gaps.

Fig. 5 shows snapshots of the electric field, $E_{z}$, obtained in vacuum, for $\mathrm{K}$ band (top) at $f=26.5 \mathrm{GHz}$ using just the antenna (Ant). The dotted
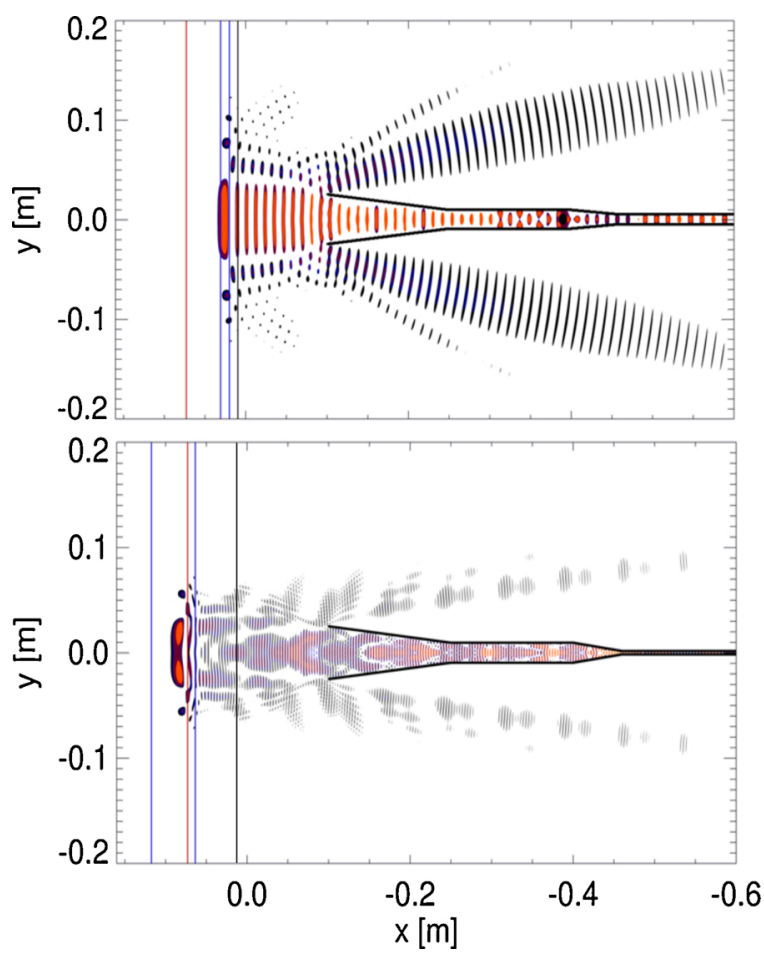

Fig. 4. Snapshots of $0^{\circ}$ simulations with a slab plasma corresponding to $\mathrm{SN}$ plasma scenario taken during the sweeps of the bands $\mathrm{K}$ (top) at a frequency of $26.5 \mathrm{GHz}$ and $\mathrm{W}$ (bottom) at $f=95 \mathrm{GHz}$. The vertical blue lines mark the isodensity region corresponding to the limits of each of the bands; the red line shows the position of the slab separatrix.
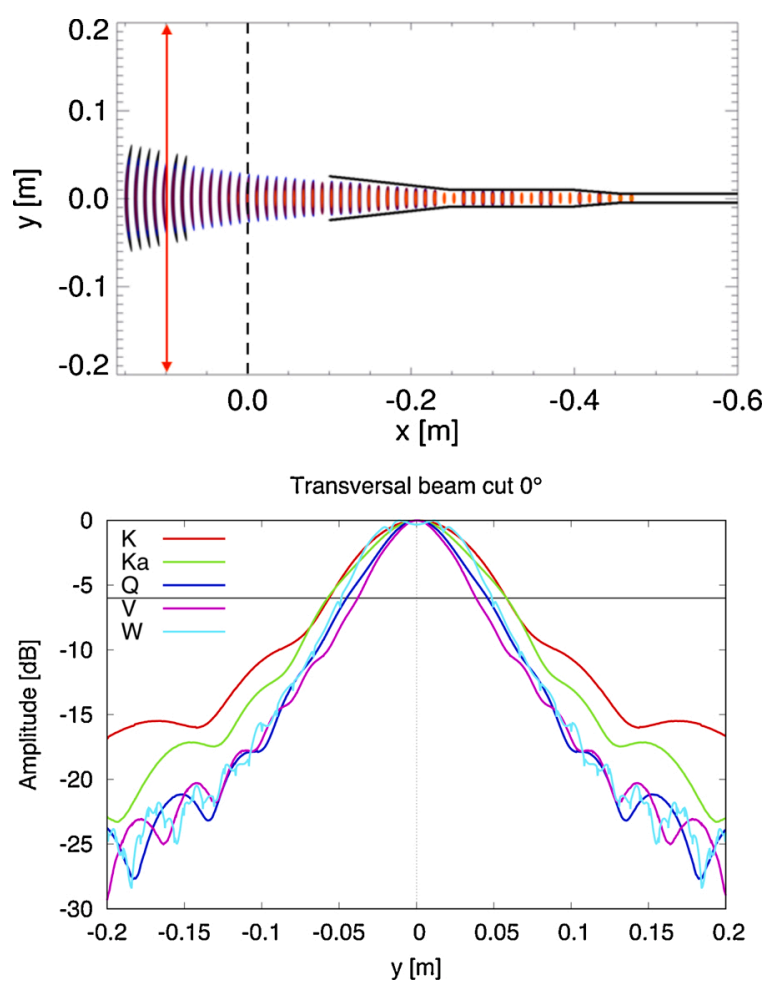

Fig. 5. Snapshots of the electric field, $E_{z}$, obtained in vacuum, for K band (top) at $f=26.5 \mathrm{GHz}$ ) using just the antenna (Ant). The red line displays the placement of the poloidal cuts and the black dashed line shows the position where the wall is placed the complete system. On the bottom, the poloidal cuts of the electric field, $E_{z}$, bottom. The intersection of the horizontal black line with the field cuts determines the HPBW. 
vertical line shows the location of the vessel wall on the full machine assembly while the red shows the placement of poloidal cuts of the electric field taken at the radial position $20 \mathrm{~cm}$ in front of the antenna mouth. The poloidal cuts of the electric field, $E_{z}$, for all the bands, appear in Fig. 5, bottom. They can be regarded as representative of the antenna radiation diagram, indicating that the antenna itself (Ant) has a radiation diagram centred at its radial axis, with a very low level of secondary lobe and a well-formed beam. The intersection of the horizontal black line with the field cuts determines the Half Power Beamwidth (HPBW).

If we take into consideration the antenna inside the tube that grants access, through the vessel wall, to the plasma, the radiation diagram is bound to be modified. For the complete structure (Ant+Cav+Wall), where the wall surface intersects the access tube perpendicularly, see Fig. 6, top. The surface of the access tubes involving the antennas acts as a non-adapted oversized waveguide and perturbs the wave pattern, originating a transversal modulation clearly visible in the transversal cuts.

These results illustrate the isolated effect of the access and wall geometry in the radiating characteristics of the antenna. The inclusion of the wall adds a reflective surface that anticipates the possibility of resonances and multi-reflections in the presence of the plasma as shown in 3.3.

\subsection{Effects of the plasma curvature and tilt}

One of the concerns of having reflectometers away from the middle plane is the influence that the curvature of the plasma, the tilt of the isodensity surface in relation to the LOS, and the existence of a poloidal
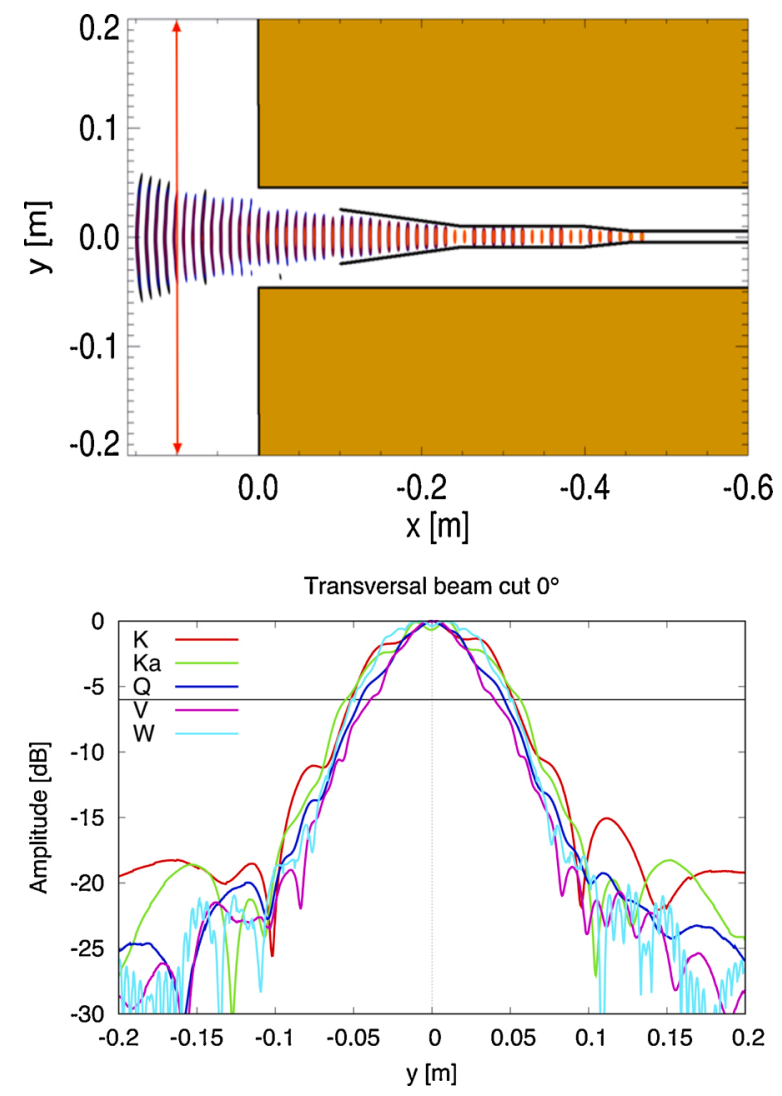

Fig. 6. Snapshot of the electric field, $E_{z}$, obtained in vacuum, for $\mathrm{K}$ band, at $f=$ $26.5 \mathrm{GHz}$, for the full structures (Ant+Cav+Wall) of gaps $0^{\circ}, 45^{\circ}$ and $-60^{\circ}$ (top). The red line displays the placement of the poloidal cuts. The poloidal cuts of the electric field, $E_{z}$, bottom, are representative of the antenna radiation diagram. The intersection of the horizontal black line with the field cuts determines the HPBW. density gradient may have on the measurements. To evaluate this point we performed simulations using just the antenna (Ant) and the plasma. In Fig. 7 we show a snapshot of a simulation of gap $0^{\circ}$, using the $\mathrm{SN}$ plasma scenario, taken during the sweeps of the band $\mathrm{K}$ at a frequency of $f=26.5 \mathrm{GHz}$.The blue lines mark the iso-density region corresponding to the limits of the band and the red line shows the position of the separatrix corresponding to a frequency of $f=87.52 \mathrm{GHz}$. This can be directly compared with Fig. 4, top, where a slab plasma was used. As can be inferred from observation, for gap $0^{\circ}$, the curvature of the plasma does not play a major role in the measurements. The error analysis comparing both situations confirms this. A similar situation occurs with the plasmas from gaps $45^{\circ}$ and $-60^{\circ}$. In these gaps, especially on the latter, the influence of curvature and tilt is felt slightly more than for gap $0^{\circ}$. Still the overall deviation from the idealised slab case is of no concern. The comparison between the error obtained from the reference case (slab) and from the SN plasma scenario shows differences lower than a few millimeters, which means that the impact of the plasma curvature and tilt on the measurements performed at these gaps is not significant. These results obtained for IDTT are in syntony with the ones obtained for DEMO in [15].

\subsection{Simulating the full system. Influence of the wall modules with plasma}

In 3.1 we looked at how the wall influences the emission/reception characteristics of the antennas and adversely reshape the probing beam. It is important to have in mind that the effective antenna to be considered consists of the aggregate comprised by the antenna and the wall. In order to fully understand the behaviour of this system, it is important to evaluate the influence of the blanket modules in the presence of the plasma since the antennas are not operating in the far field region and, therefore, the antennas cannot be considered separated from the target (plasma). In Fig. 8, we present snapshots of simulations of gap $0^{\circ}, 45^{\circ}$ and $-60^{\circ}$ using the $\mathrm{SN}$ plasma scenario, taken during the sweep of the $\mathrm{W}$ bands at $f=95 \mathrm{GHz}$. The vertical blue lines mark the iso-density region corresponding to the limits of the band; the red line shows the position of the separatrix corresponding to a frequency of $f=87.52 \mathrm{GHz}$. We can have a qualitative picture on how the different elements of complexity add up to the final result by comparing the behaviour of gap $0^{\circ}$ in the ideal slab case (Fig. 4), with the introduction of plasma curvature (Fig. 7) and, finally, with the full structure surrounding the antenna (Fig. 8). The full structure with the curve and tilt of plasma introduces a visible distortion of the field patterns with the appearance of resonances in the lower $\mathrm{K}$ and $\mathrm{Ka}$ bands passing to a regime of multi-reflections at higher frequency bands. These effects are less severe at gap $0^{\circ}$ and more pronounced for gap $-60^{\circ}$. For the lower frequencies, we notice the formation of a resonant field structure just in front of the gap between

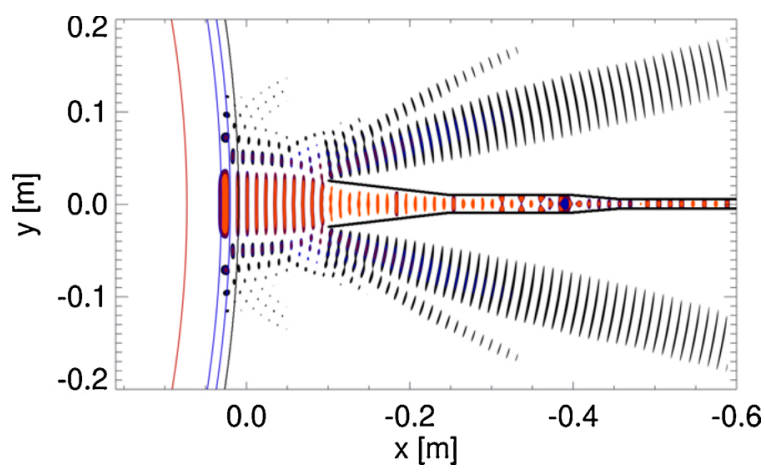

Fig. 7. Snapshots of $0^{\circ}$ simulations with a plasma corresponding to SN plasma scenario, using just the antenna (Ant), taken during the sweep of the bands $\mathrm{K}$ at a frequency of $26.5 \mathrm{GHz}$. The blue lines mark the iso-density region corresponding to the limits of each of the bands; the red line shows the position of the slab separatrix. 

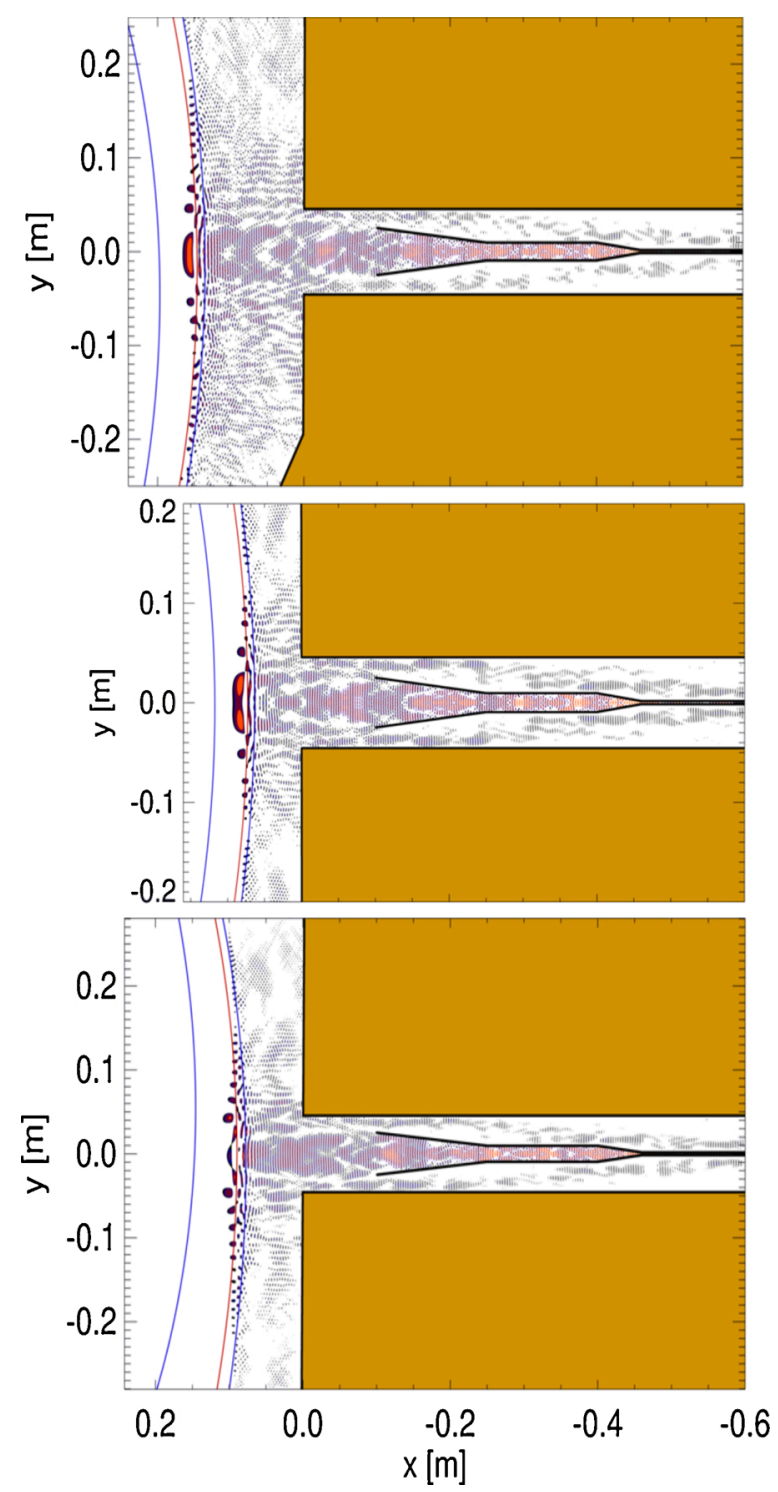

Fig. 8. Snapshots of gaps $45^{\circ}, 0^{\circ}$ and $-60^{\circ}$ simulations with a plasma corresponding to SN plasma scenario, using the complete system (Ant+Cav+Wall), taken during the sweep of the band $\mathrm{W}$ at $f=95 \mathrm{GHz}$. The blue lines mark the iso-density region corresponding to the limits of the band; the red line shows the position of the separatrix.

the wall and the plasma cut-off layer, and also within the access tube encircling the antennas. For the higher frequencies, the resonant conditions are not met, and the main negative effect comes from the many multiple reflections formed between the wall and the plasma reflection layer. Inside this cavity-like region it is possible to observe the existence of multiple signal paths leading back to the antenna. That said, it is also possible to infer that albeit these adverse effects the field structure maintains its main characteristics and the phase is not significantly affected. Consequently measurements can be performed with errors within the requirements as shown below.

\subsection{Assessment of the measurement performance}

The primary role of the IDTT PPR system is to provide real-time measurements of the density profile covering the edge plasma region up to and just inside the magnetic separatrix for relevant operating scenarios. The IDTT Plasma Control System (PCS) will then estimate the radial position of the magnetic separatrix from the measured profiles and use it to estimate and track the distance (gap) between its position and the wall. We should note that the PCS might end-up using a different definition for the gap distance that uses a density layer other than the one at the magnetic separatrix. For known regimes of operation, the evolution of the gap can signal the approach of the plasma to the first wall with enough time for the PCS to take preventive action. This procedure assumes that the nominal separatrix density is known for each regime of operation. The measurement requirements of a PPR system specify that the gap between the plasma and the first wall shall be measured with a typical maximum error of $\pm 1 \mathrm{~cm}$. Since the position of the wall will be known with great accuracy from the metrological surveys of the vacuum vessel, this means that the position of the magnetic separatrix needs to be determined from the density profiles provided by the PPR system within the requirements. Therefore, in assessing the performance of the PPR in-vessel systems, we will use the error in the determination of the separatrix position from the reconstructed density profiles as a quantitative indication of how well these systems will perform during operation.

\subsection{Data processing}

The synthetic reflectometers of the different gaps are excited with a frequency-modulated (FM) signal, having an instantaneous frequency, $f(t)=f_{c}+f_{\Delta} g(t)$, with $f_{\Delta}<f_{c}$, where $f_{\Delta}$ is the frequency deviation, $f_{c}$ is the carrier frequency and $g(t)$ the modulating signal. In our case a ramp $g(t)=t / T_{s}$, where $T_{s}$ is the duration of a single sweep. During the sweep, the instantaneous frequency of the signal varies from a minimum frequency $f_{m}=f_{c}$ to a maximum frequency $f_{M}=f_{c}+f_{\Delta}$. Therefore, the signal instantaneous phase is given by:

$\phi(t)=2 \pi f_{c} t+2 \pi f_{\Delta} \int_{0}^{t} g(\lambda) \mathrm{d} \lambda=2 \pi f_{c} t+\pi f_{\Delta} t^{2} / T_{s}$.

The input FM signal is given by:

$i(t)=\cos \phi(t)$.

This signal propagates along the (numerical) plasma and is reflected back to the antenna. The detected signal, $d(t)$, has a phase difference $\varphi(t)$ in respect to Eq. (4) that conveys information about the wave propagation in the waveguide, vacuum and plasma:

$d(t)=\cos [\phi(t)+\varphi(t)]$.

Multiplying the detected signal $d(t)$ with in-phase $[\sin \phi(t)]$ and quadrature $[\cos \phi(t)]$ samples of the input signal $\mathrm{i}(\mathrm{t})$ we get:

$$
\begin{aligned}
& m_{I}(t)=d(t) \sin \phi(t)=\frac{1}{2} \sin [2 \phi(t)+\varphi(t)]+\frac{1}{2} \sin \varphi(t) \\
& m_{Q}(t)=d(t) \cos \phi(t)=\frac{1}{2} \cos [2 \phi(t)+\varphi(t)]+\frac{1}{2} \cos \varphi(t)
\end{aligned}
$$

From Eq. (6), we observe that $m_{I}(t)$ and $m_{Q}(t)$ have both high frequency components (twice as high as the excitation signals) and low frequency ones, which carry the information about $\varphi(t)$. Applying low pass filtering we obtain these low frequency components:

$s_{I}(t)=m_{I}(t) * h_{B}(t)=\sin \varphi(t)$

$s_{Q}(t)=m_{Q}(t) * h_{B}(t)=\cos \varphi(t)$,

where $h_{B}(t)$ is the impulsive response of a Butterworth low-pass filter and $*$ denotes the convolution operation. The signals $s_{I}(t)$ and $s_{Q}(t)$ are the so-called in-phase and quadrature reflectometry signals. An I/Q temporal technique is used to evaluate the phase:

$\varphi(t)=\tan ^{-1}\left[\frac{s_{I}(t)}{s_{Q}(t)}\right]$

The phase derivative $\partial \varphi / \partial f$ is evaluated from $\varphi(t)$ using a numerical 
differentiation procedure. In Fig. 9, top, the phase derivative evaluated for the SN plasma scenario for gap $-60^{\circ}$ is represented against the theoretical Wentzel-Kramers-Brillouin-Jeffreys (WKBJ) phase derivative, directly calculated from the input data. The vertical red line marks the separatrix frequency. The vertical shaded areas denote the frequency ranges of the different bands.

The phase derivative allows us to calculate the position of a given reflecting layer $r\left(n_{e}\right)$, and recover the electronic density profile using [38]

$r(F)=\frac{c}{2 \pi^{2}} \int_{0}^{F} \frac{\partial \varphi}{\partial f} \frac{\mathrm{d} f}{\sqrt{F^{2}-f^{2}}}$.

The profile reconstructed from the phase derivatives, for the SN plasma scenario, gap $-60^{\circ}$, appears in Fig. 9, bottom. The horizontal red line marks the separatrix density. The vertical shaded band shows the error tolerance of $\pm 1 \mathrm{~cm}$ in the position of the separatrix.

The error in the reflectometry measurement of a given position in the plasma can be evaluated as:

$\operatorname{Error}(F)=\frac{c}{2 \pi^{2}} \int_{0}^{F}\left(\frac{\partial \varphi}{\partial f}-\frac{\partial \varphi_{\mathrm{WKBJ}}}{\partial f}\right) \frac{\mathrm{d} f}{\sqrt{F^{2}-f^{2}}}$,

where $\partial \varphi / \partial F$ is the phase derivative of the measurement and $\partial \varphi_{\mathrm{WKBJ}} / \partial F$ is the phase derivative of the reference measurement. At the separatrix, the error is evaluated using Eq. (10) at the cut-off frequency corresponding to the electronic density at the separatrix, that is:

Error $_{\text {sep }}=\operatorname{Error}\left[F\left(n_{e_{\text {sep }}}\right)\right]$.
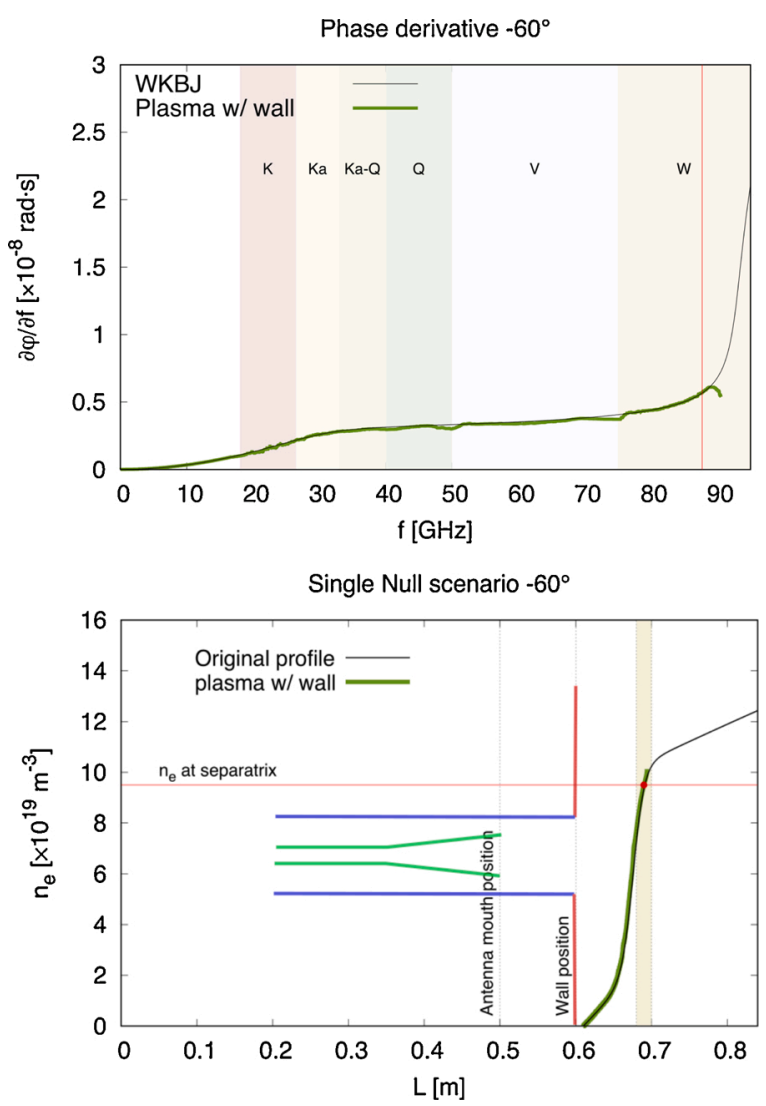

Fig. 9. On top, the phase derivative for the SN plasma scenario. The vertical red line marks the separatrix frequency. The vertical shaded areas denote the frequency ranges of the different bands. On the bottom, the reconstructed density profiles for the SN plasma scenario. The horizontal red line marks the separatrix density. The vertical shaded band shows the error tolerance of $\pm 1 \mathrm{~cm}$ in the position of the separatrix. Superimposed is a schematic of the waveguide/ antenna and wall structure visually guiding the positioning of the profile.
Fig. 10 depicts the evolution of the measurement error as a function of the probing frequency obtained for SN scenario, for the 3 gaps considered. The horizontal band depicts the maximum error of $\pm 1 \mathrm{~cm}$ specified in the PPR measurement requirements, while the vertical lines indicate the separatrix plasma frequency. Apart from the real configuration with the full assembly (Ant $+\mathrm{Cav}+$ Wall) we also include the academic comparison cases of a slab plasma (with and without wall) and plasma without wall. The qualitative inference made above is confirmed by the quantitative analysis of the error. We observe that the influence of the wall and plasma curvature on the LFS gaps is not a major problem since the error is well within the error tolerance. The error is lower at gap $0^{\circ}$ and more pronounced at gap $-60^{\circ}$, although we notice that the introduction of the wall impacts gap $45^{\circ}$ around the Ka band.
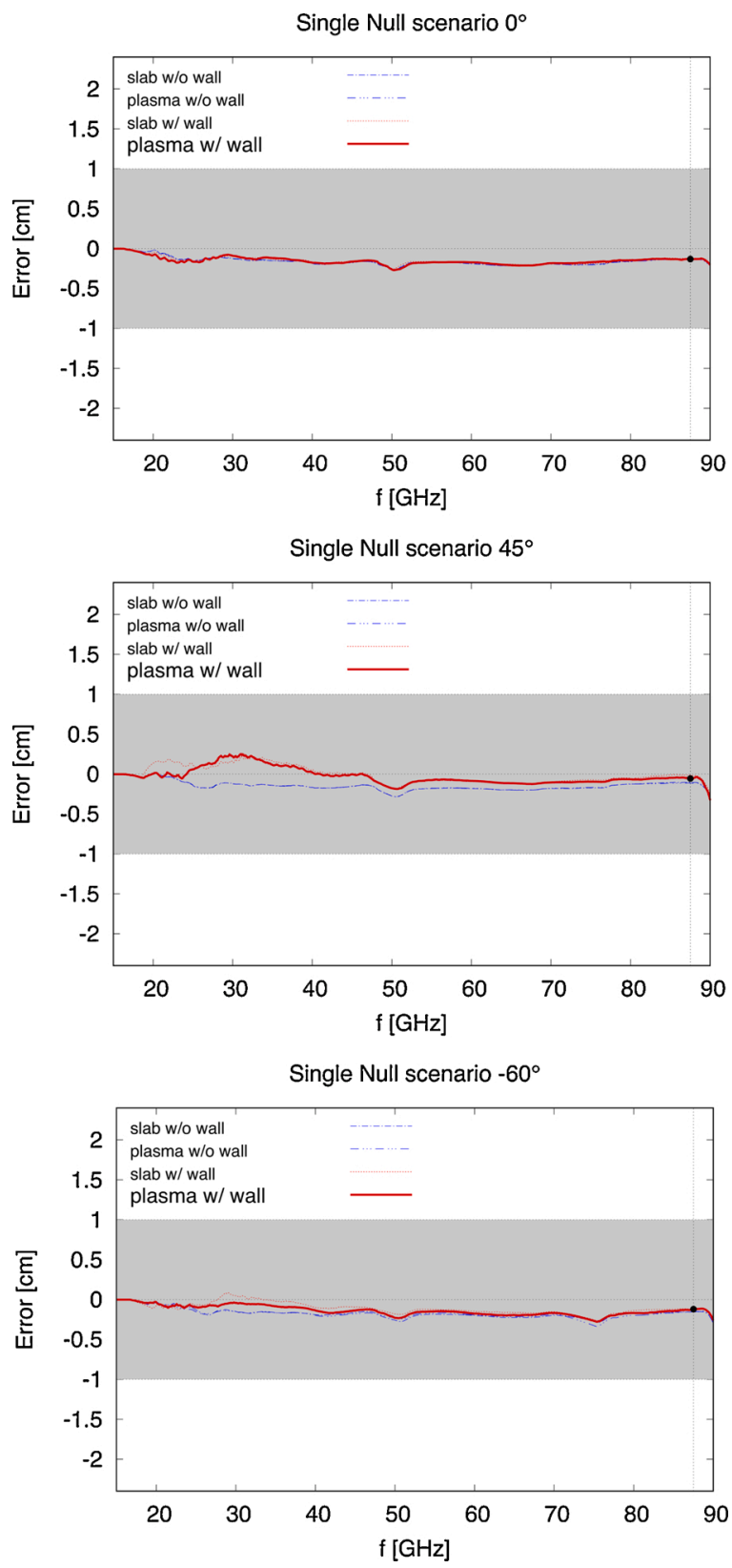

Fig. 10. Evolution of the measurement error with the probing frequency for the 3 gaps in study for the SN scenario case. The horizontal band depicts the maximum error of $\pm 1 \mathrm{~cm}$ specified in the PPR measurement requirements, while the vertical lines indicate the separatrix plasma frequency. 


\subsection{Double null scenario with the single null antenna alignment}

In this section we will show the results obtained from the full wave simulations using a DN plasma scenario. The alignment of the antennas was the same as the one used above for the SN scenario (DNsn). A key question to answer is if this optimization, done for the SN scenario, still allows good results to be obtained in the DN scenario. This is important because it guarantees that the same hardware setup can cope with different conditions. The same procedures used to treat the SN scenario were deployed with the DNsn. A comparison of the results obtained with the DNsn and SN is presented in Fig. 11. The evolution of the measurement errors with the probing frequency, for the 3 gaps in study, are compared between the DN scenario with the antennas aligned for the SN scenario (DNsn), and the SN scenario case. The horizontal band depicts
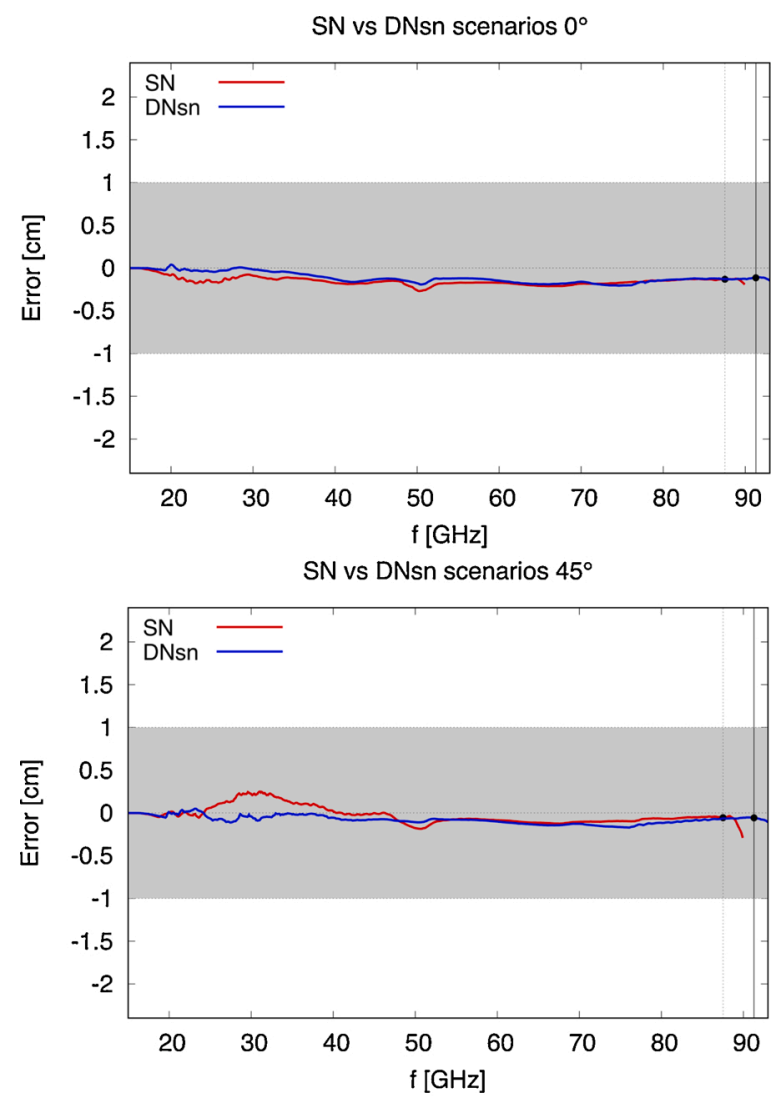

SN vs DNsn scenarios $-60^{\circ}$

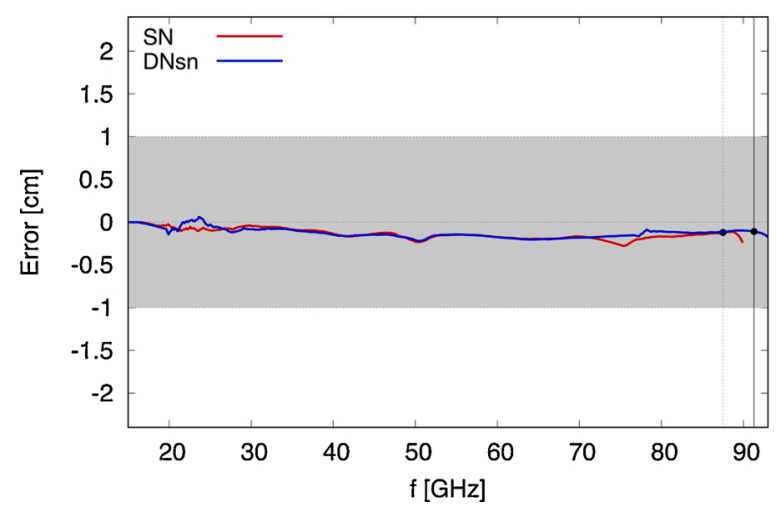

Fig. 11. Comparison of the evolution of the measurement errors with the probing frequency for the 3 gaps in study for the DN scenario with the antennas aligned for the SN scenario (DNsn) and for the SN scenario case. The horizontal band depicts the maximum error of $\pm 1 \mathrm{~cm}$ specified in the PPR measurement requirements, while the vertical lines indicate the separatrix plasma frequency. the maximum error of $\pm 1 \mathrm{~cm}$ specified in the PPR measurement requirements, while the vertical lines indicate the separatrix plasma frequency. The results are very positive since there is no practical difference between the position errors in the two cases in the LFS gaps. This is of paramount importance as it means that the tentative optimization tried for the SN plasma scenario is robust enough to respond to the DN scenario. Looking at the comparative results, it seems, paradoxically, at $\mathrm{K}$ band on gap $0^{\circ}$, and more visible around Ka band on gap $45^{\circ}$, the error is slightly better for the DNsn. This fact has not to do with the optimisation of the LOS but with the particular resonance conditions that appear with the wall-plasma ensemble at this lower bands, which are more effective in the SN scenario. This gives indication that the tilted angle of the probing wave when compared to the a probing normal to the isodensity surface plays a small role for the scenarios evaluated.

\section{Conclusions}

Three synthetic reflectometers have been set up on the LFS; the first (gap $0^{\circ}$ ) on the equatorial plane, the second on the upper part of the torus (gap $45^{\circ}$ ) and the third on the lower part of the torus (gap $-60^{\circ}$ ); and their performance for the selected IDTT scenarios assessed. The first step to implement them consisted in revising the input data (plasma scenarios), complementing it according to a model and preparing the inputs necessary for REFMULF. These are necessary steps that bring together the available data set, many times incomplete, and the theoretical models needed to complement it with educated models based on known theory or experimental data. The reasoning and procedure exposed here for the PPR simulation using REFMULF and IDDT data as inputs are similar to the ones adopted for the simulations of ITER [39] and DEMO PPRs [15].

For the antennas and access to the plasma we resorted to one of the tentative layouts adopted in DEMO studies [40]. Concerning the effects the structures surrounding the antennas have on the measurements of the reflectometers at each gap, we can concluded that the design tested does not have a significant impact on the performance of the reflectometers, and can therefore be considered a safe design. This structure design can also provide thermal and erosion protection. The alignment of the antennas, optimised for the SN scenario, proved to be equally efficient for the DN scenario. The impact of probing the plasma away from the midplane does not impose major difficulties for the three gaps implemented.

Simulations of the full systems, which include the effects of the plasma and structure and their interplay, confirms a good performance for the LFS reflectometers, gaps $0^{\circ}, 45^{\circ}$ and $-60^{\circ}$ with measurement errors bars well within the $\pm 1 \mathrm{~cm}$ error requirements, and with deviations of the order of some millimeters at the separatrix.

It was shown for DEMO that the edge turbulence has no significant effects on position measurements [15]. However, since for IDTT, a different turbulence wavenumber spectrum is expected, the real impact of turbulence should be evaluated. Unfortunately, at this stage of IDTT design, no information is available on the $2 \mathrm{D}$ poloidal spectral characteristics of turbulence (as a function of the wave number) and radial profile of turbulence level. This assessment on the impact of turbulence in the measurements of the in-vessel PPR systems will be the object of future work, using IDTT turbulence scenarios if they become available, or resorting to consistent models as it has been done for ITER [39] and DEMO [41].

Although the planed reference scenario for IDTT is a SN scenario at 5.5 MA, the machine aims at being extremely flexible, allowing for additional alternative divertor concepts and plasma magnetic configurations to be tested such as the Double Null (DN), the SnowFlake (SF), the X-divertor, negative triangularity (DN and SN)and the Double Super$\mathrm{X}$ [21]. We started looking at the SN and DN configurations but this study must be complemented in coming ones with the remaining possible scenarios. It is important to see which of the proposed PPRs and chosen LOS still deliver good results with scenarios that can be 
significantly different from SN and DN.

The main conclusion to take from this exercise is that the proposed positions (or any small variations) are suitable for the placement of reflectometry systems at IDTT. The results encourage efforts towards an actual implementation of such systems for IDTT. Even a reduced system having just gap $0^{\circ}$ and one of the other two gaps, $45^{\circ}$ or $-60^{\circ}$, would be a major step for reflectometry in general, and for IDDT and DEMO in particular, since a profile measuring system away from the midplane has never been tested before. It would consist in one of the major advances in the last two decades, and we dare to say, with a major impact on DEMO reflectometry plans.

\section{Declaration of Competing Interest}

The authors report no declarations of interest.

\section{Acknowledgments}

This work has been carried out within the framework of the EUROfusion Consortium and has received funding from the EURATOM research and training programme 2014-2018 and 2019-2020 under grant agreement No 633053 and within the framework of the French Federation for Magnetic Fusion Studies (FR-FCM). IST activities also received financial support from Fundação para a Ciência e Tecnologia through project UID/FIS/50010/2019. The views and opinions expressed herein do not necessarily reflect those of the European Commission. Simulations were made using EUROfusion High Performance Computer Marconi, under project DTTsimul.

\section{References}

[1] R. Albanese, on behalf of the WPDTT2 Team and the DTT Project Proposa Contributors, DTT: a divertor tokamak test facility for the study of the powe exhaust issues in view of DEMO, Nucl. Fusion 57 (1) (2016) 016010, https://doi. org/10.1088/0029-5515/57/1/016010.

[2] G. Marchiori, G. De Masi, R. Cavazzana, A. Cenedese, N. Marconato, R. Moutinho, A. Silva, EUROfusion-IM team, study of a plasma boundary reconstruction method based on reflectometric measurements for control purposes, IEEE Trans. Plasma Sci. 46 (5) (2018) 1285, https://doi.org/10.1109/TPS.2018.2797549.

[3] F. Simonet, Measurement of electron density profile by microwave reflectometry on tokamaks, Rev. Sci. Instrum. 56 (5) (1985) 664, https://doi.org/10.1063/ 1.1138200.

[4] H. Bottollier-Curtet, G. Ichtchenko, Microwave reflectometry with the extraordinary mode on tokamaks: determination of the electron density profile of Petula-B, Rev. Sci. Instrum. 58 (4) (1987) 539, https://doi.org/10.1063/ 1.1139266.

[5] E. Mazzucato, R. Nazikian, Radial scale length of turbulent fluctuations in the main core of TFTR plasmas, Phys. Rev. Lett. 71 (1993) 1840, https://doi.org/10.1103/ PhysRevLett.71.1840.

[6] E. Gusakov, S. Heuraux, A. Popov, M. Schubert, Reconstruction of the turbulence radial profile from reflectometry phase root mean square measurements, Plasma Phys. Control. Fusion 54 (4) (2012) 045008, https://doi.org/10.1088/0741-3335/ 54/4/045008.

[7] E.V. Sysoeva, F. da Silva, E.Z. Gusakov, S. Heuraux, A.Y. Popov, Electron cyclotron resonance heating beam broadening in the edge turbulent plasma of fusion machines, Nucl. Fusion 55 (3) (2015) 033016, https://doi.org/10.1088/0029 5515/55/3/033016.

[8] F. Clairet, S. Heuraux, C. Bottereau, D. Molina, L. Ducobu, F. Leroux, A. Barbuti, Fast sweeping reflectometry upgrade on Tore Supra, Rev. Sci. Instrum. 81 (10) (2010) 10D903, https://doi.org/10.1063/1.3460624.

[9] G.V. Zadvitskiy, S. Heuraux, C. Lechte, S. Hacquin, R. Sabot, Edge turbulence effect on ultra-fast swept reflectometry core measurements in tokamak plasmas, Plasma Phys. Control. Fusion 60 (2) (2018) 025025, https://doi.org/10.1088/1361-6587/ aa9807.

[10] O.L. Krutkin, A.B. Altukhov, A.D. Gurchenko, E.Z. Gusakov, S. Heuraux, M. A. Irzak, L.A. Esipov, T.P. Kiviniemi, C. Lechte, S. Leerink, Investigation of nonlinear effects in Doppler reflectometry using full-wave synthetic diagnostics, Plasma Sci. Technol. 22 (6) (2020) 064001, https://doi.org/10.1088/2058-6272/ ab5c28.

[11] R.B.- Morales, S. Heuraux, R. Sabot, S. Hacquin, F. Clairet, the Tore Supra Team, Reconstruction of hollow areas in density profiles from frequency swept reflectometry, Plasma Sci. Technol. 22 (6) (2020) 064005, https://doi.org/ 10.1088/2058-6272/ab7b5a.

[12] R.B.- Morales, S. Hacquin, S. Heuraux, R. Sabot, New density profile reconstruction methods in X-mode reflectometry, Rev. Sci. Instrum. 88 (4) (2017) 043503-043511, https://doi.org/10.1063/1.4979513.
[13] R. Sabot, F. Clairet, G.D. Conway, L. Cupido, X. Garbet, G. Falchetto, T. Gerbaud, S. Hacquin, P. Hennequin, S. Heuraux, C. Honoré, G. Leclert, L. Menese, A. Sirinelli, L. Vermare, A. Truc, Recent results on turbulence and MHD activity achieved by reflectometry, Plasma Phys. Control. Fusion 48 (12B) (2006) B421, https://doi.org/10.1088/0741-3335/48/12B/S40.

[14] T. Estrada, K. Nagasaki, E. Blanco, G. Perez, V. Tribaldos, Microwave reflectometry diagnostics: present day systems and challenges for future devices, Plasma Fusion Res. 7 (2012) 2502055, https://doi.org/10.1585/pfr.7.2502055.

[15] E. Ricardo, F. da Silva, S. Heuraux, A. Silva, Assessment of a multi-reflectometers positioning system for DEMO plasmas, J. Instrum. 14 (8) (2019) C08010, https:// doi.org/10.1088/1748-0221/14/08/C08010.

[16] J. Santos, L. Guimarãis, M. Zilker, W. Treutterer, M. Manso, A.S.D.E.X. the, Upgrade Team, Reflectometry-based plasma position feedback control demonstration at ASDEX Upgrade, Nucl. Fusion 52 (3) (2012) 032003, https://doi. org/10.1088/0029-5515/52/3/032003.

[17] J.M. Santos, L. Guimarãis, C. Rapson, G. Santos, A. Silva, W. Treutterer, M. Zilker, ASDEX Upgrade Team, EUROfusion MST1 Team, Real-time reflectometry - an ASDEX Upgrade DCS plugin App for plasma position and shape feedback control, Fusion Eng. Des. 123 (2017) 593, https://doi.org/10.1016/j. fusengdes.2017.03.016.

[18] R. Martone, R. Albanese, F. Crisanti, P. Martin, A. Pizzuto, DTT Divertor Tokamak Test facility - Interim Design Report, ENEA Italian Agency for New Technologies, Energy and Sustainable Economic Development, 2019 April.

[19] PEX/DTTshared folder in the DTT, https://eneabox-dtt.enea.it/index.ph $\mathrm{p} / \mathrm{s} /$ fDUYNaRt5XRVX7i (password protected).

[20] EUROfusion IDM, https://idm.euro-fusion.org/?uid=2NZJH6 (password protected).

[21] R. Ambrosino, R. Albanese, G. Calabrò, A. Castaldo, F. Crisanti, V.P. Loschiavo, M. de Magistris, S. Minucci, G. Ramogida, The DTT device: poloidal field coil assessment for alternative plasma configurations, Fusion Eng. Des. 122 (2017) 322, https://doi.org/10.1016/j.fusengdes.2017.01.055.

[22] L. Frassinetti, M.N.A. Beurskens, S. Saarelma, J.E. Boom, E. Delabie, J. Flanagan, M. Kempenaars, C. Giroud, P. Lomas, L. Meneses, C.S. Maggi, S. Menmuir, I. Nunes, F. Rimini, E. Stefanikova, H. Urano, G. Verdoolaege, J.E.T. Contributors, Global and pedestal confinement and pedestal structure in dimensionless collisionality scans of low-triangularity H-mode plasmas in JET-ILW, Nucl. Fusion 57 (1) (2017) 016012, https://doi.org/10.1088/0029-5515/57/1/016012.

[23] ITER physics expert group on diagnostics and ITER physics basis editors, Chapter 7: measurement of plasma parameters, Nucl. Fusion 39 (12) (1999) 2541, https://doi. org/10.1088/0029-5515/39/12/307.

[24] W. Biel, R. Albanese, R. Ambrosino, M. Ariola, M.V. Berkel, I. Bolshakova, K. J. Brunner, R. Cavazzana, M. Cecconello, S. Conroy, A. Dinklage, I. Duran, R. Dux, T. Eade, S. Entler, G. Ericsson, E. Fable, D. Farina, L. Figini, C. Finotti, Th. Franke, L. Giacomelli, L. Giannone, W. Gonzalez, A. Hjalmarsson, M. Hron, F. Janky, A. Kallenbach, J. Kogoj, R. K\&ldquo;onig, O. Kudlaceg, R. Luis, A. Malaquias, O. Marchuk, G. Marchiori, M. Mattei, F. Maviglia, G. De Masi, D. Mazon, H. Meister, K. Meyer, D. Micheletti, S. Nowak, Ch. Piron, A. Pironti, N. Rispoli, V. Rohde, G. Sergienko, S. El Shawish, M. Siccinio, A. Silva, F. da Silva, C. Sozzi, M. Tardocchi, M. Tokar, W. Treutterer, H. Zohm, Diagnostics for plasma control from ITER to DEMOFusion Eng. Des. 146 (2019) 465, https://doi.org/10.1016/j fusengdes.2018.12.092.

[25] F. da Silva, S. Heuraux, E. Ricardo, A. Silva, T. Ribeiro, Benchmarking 2D against 3D FDTD codes in the assessment of reflectometry performance in fusion devicesJ. Instrum. 14 (2019), https://doi.org/10.1088/1748-0221/14/08/C08004.

[26] F. da Silva, S. Heuraux, E. Ricardo, P. Quental, J. Ferreira, Assessment of the measurement performance of the in-vessel system of gap 6 of the ITER plasma position reflectometer using a finite-difference time-domain Maxwell full-wave codeRev. Sci. Instrum. 87 (11) (2016) 11E727, https://doi.org/10.1063/ 1.4962356

[27] F. da Silva, S. Heuraux, S. Hacquin, M. Manso, Unidirectional transparent signal injection in finite-difference time-domain electromagnetic codes - application to reflectometry simulationsJ. Comput. Phys. 203 (2) (2005) 467, https://doi.org/ 10.1016/j.jcp.2004.09.002.

[28] P. Varela, A. Silva, J.H. Belo, Testing of the ITER plasma position reflectometry high-field side in-vessel antenna assembly prototypeRev. Sci. Instrum. 89 (10) (2018) 10H102, https://doi.org/10.1063/1.5036743.

[29] P. Varela, J.H. Belo, A. Silva, F. da Silva, Design status of the in-vessel subsystem of the ITER plasma position reflectometry systemJ. Instrum. 14 (09) (2019) C09002, https://doi.org/10.1088/1748-0221/14/09/C09002.

[30] L. Xu, N. Yuan, FDTD formulations for scattering from 3-d anisotropic magnetized plasma objectsIEEE Antennas Wirel. Propag. Lett. 5 (2006) 335, https://doi.org/ 10.1109/LAWP. 2006.878901.

[31] F. da Silva, S. Heuraux, E. Ricardo, A. Silva, T. Ribeiro, Modelling reflectometry diagnostics: finite-difference time-domain simulation of reflectometry in fusion plasmasJ. Instrum. 14 (2019) C08003, https://doi.org/10.1088/1748-0221/14/ 08/C08003.

[32] J.-P. Bérenger, A perfectly matched layer for the absorption of electromagnetic wavesJ. Comput. Phys. 114 (2) (1994) 185, https://doi.org/10.1006/ jcph.1994.1159.

[33] P. Varela, M.E. Manso, A. Silva, J. Fernandes, F. Silva, Initialization of plasma density profiles from reflectometryRev. Sci. Instrum. 66 (10) (1995) 4937, https:// doi.org/10.1063/1.1146178.

[34] T.J. Boyd, J.J. Sanderson, The Physics of Plasmas, Chapter 11, Cambridge University Press, 2003. ISBN-13 978-0521459129.

[35] C. Fanack, I. Boucher, F. Clairet, S. Heuraux, G. Leclert, X.L. Zou, Ordinary-mode reflectometry: modification of the scattering and cut-off responses due to the shape 
of localized density fluctuationsPlasma Phys. Control. Fusion 38 (11) (1996) 1915 https://doi.org/10.1088/0741-3335/38/11/004.

[36] C.A. Balanis, Antenna Theory: Analysis and Design, 4th ed., John Wiley and Sons Inc., 2016. ISBN: 9781118642061.

[37] P. Varela, J.H. Belo, P.B. Quental, Performance assessment of the antenna setup for the ITER plasma position reflectometry in-vessel systemsRev. Sci. Instrum. 87 (11) (2016) 11E713, https://doi.org/10.1063/1.4961291.

[38] I.H. Hutchinson, Principles of Plasma Diagnostics, 2nd ed., Cambridge University Press, 2005, p. 143. ISBN: 13 978-0521675741.
[39] F. da Silva, Assessment of the measurement performance of the PPR (D13), 2018. F4E-FPA-375 (DG): SG04, Fusion for Eneroy DMS reference: F4E D 24V2MW.

[40] A. Silva, DC-4-T005-D001 Final Report on DEMO Diagnostic R\&D: Microwave Diagnostics (IST), 2016. Eurofusion IDM reference: EFDA_D_2MX6B6 v1.0.

[41] E. Ricardo, F. da Silva, S. Heuraux, A. Silva, The effect of plasma turbulence on the plasma position reflectometry measurements at DEMO, Fusion Eng. Des., 2021 (submitted for publication). 